PROCEEDINGS OF THE

AMERICAN MATHEMATICAL SOCIETY

Volume 140, Number 1, January 2012, Pages 167-171

S 0002-9939(2011)11118-3

Article electronically published on May 18, 2011

\title{
ON COMMUTATIVITY OF THE COMMUTANT OF STRONGLY IRREDUCIBLE OPERATOR
}

\author{
JUE-XIAN LI \\ (Communicated by Richard Rochberg)
}

\begin{abstract}
In 2006, C. L. Jiang and Z. Y. Wang posed an open problem: If $T$ is a strongly irreducible operator, is $\mathcal{A}^{\prime}(T) / \operatorname{rad} \mathcal{A}^{\prime}(T)$ commutative? They conjectured that the answer is positive. In this paper, to negatively answer their problem, a counterexample is given.
\end{abstract}

\section{INTRODUCTION}

Let $\mathcal{H}$ be a complex separable Hilbert space, and let $\mathcal{B}(\mathcal{H})$ denote the Banach algebra of all bounded linear operators acting on $\mathcal{H}$. For $T \in \mathcal{B}(\mathcal{H})$, let $\mathcal{A}^{\prime}(T)$ and $\sigma(T)$ denote the commutant and the spectrum of $T$, respectively. Letting $\mathcal{A}$ be a unital Banach algebra, we write $\operatorname{rad} \mathcal{A}$ for the Jacobson radical of $\mathcal{A}$, i.e.

$$
\operatorname{rad} \mathcal{A}=\{A \in \mathcal{A}: \sigma(A B)=\sigma(B A)=\{0\}, \forall B \in \mathcal{A}\} .
$$

An operator $T$ in $\mathcal{B}(\mathcal{H})$ is called strongly irreducible, denoted by $T \in S I$, if $\mathcal{A}^{\prime}(T)$ does not include any nontrivial idempotent operators. Obviously, strong irreduciblity of an operator is invariant under similarity sense. Moreover, it is shown in [4] and 5] that the class of such operators is a suitable replacement of the Jordan blocks in $\mathcal{B}(\mathcal{H})$.

When $\mathcal{H}$ is a finite dimensional space, from the Jordan Canonical Form Theorem, $T$ in $\mathcal{B}(\mathcal{H})$ is strongly irreducible if and only if there exists a basis of $\mathcal{H}$ such that the representing matrix of $T$ with respect to this basis is a Jordan block:

$$
J(\lambda)=\left(\begin{array}{cccc}
\lambda & 1 & & 0 \\
& \ddots & \ddots & \\
& & \lambda & 1 \\
0 & & & \lambda
\end{array}\right) .
$$

It is easily known that $\mathcal{A}^{\prime}(J(\lambda))$ consists of all upper-triangular matrices as follows:

$$
A=\left(\begin{array}{cccc}
a_{1} & a_{2} & \cdots & a_{k} \\
& \ddots & \ddots & \vdots \\
& & a_{1} & a_{2} \\
0 & & & a_{1}
\end{array}\right) .
$$

Received by the editors October 28, 2010.

2010 Mathematics Subject Classification. Primary 47B37; Secondary 47C05.

Key words and phrases. Strongly irreducible operator, operator weighted shifts.

This project is supported by the National Natural Science Foundation of China (No. 10971079).

(c)2011 American Mathematical Society Reverts to public domain 28 years from publication 
As a result, $\mathcal{A}^{\prime}(J(\lambda))$ is exactly commutative. However, if $\mathcal{H}$ is an infinite dimensional space, an example was given in [3] to show that there exists a strongly irreducible operator such that its commutant is not commutative. Further, C. L. Jiang and Z. Y. Wang posed the following problem (cf. [5], p. 147):

Open problem JW. If $T \in S I$, is $\mathcal{A}^{\prime}(T) / \operatorname{rad} \mathcal{A}^{\prime}(T)$ commutative?

Moreover, they [5] showed that the operator class

$$
\left\{T \in S I: \mathcal{A}^{\prime}(T) / \operatorname{rad} \mathcal{A}^{\prime}(T) \text { is commutative }\right\}
$$

is dense in the set of strongly irreducible operators in the norm topology. Also, Jiang 22 proved that if $T$ is a Cowen-Douglas operator and $T \in S I$, then $\mathcal{A}^{\prime}(T) / \operatorname{rad} \mathcal{A}^{\prime}(T)$ is commutative. Based on these conclusions, they conjectured that the answer to the above problem is positive ([5], p. 85).

In this paper, a counterexample is given to negatively answer this problem.

\section{Preliminaries}

A generalization of a nilpotent Jordan block in an infinite dimensional space is a weighted shift operator. However, the commutant of a weighted shift is also commutative [8]. In view of this fact, we consider the following shifts with operator weights introduced by A. L. Lambert in [6].

Let $\mathbb{C}^{n}$ be an $n$-dimensional complex Hilbert space and let $\mathcal{H}=\bigoplus_{k=0}^{\infty} \mathbb{C}^{n}$. Suppose that $\left\{W_{k}\right\}_{k=1}^{\infty}$ is a sequence of operators in $\mathcal{B}\left(\mathbb{C}^{n}\right)$ such that each $W_{k}$ is invertible and $\sup _{k}\left\{\left\|W_{k}\right\|\right\}<\infty$. Call

$$
S=\left(\begin{array}{cccccc}
0 & W_{1} & & & & \\
& 0 & W_{2} & & 0 & \\
& & \ddots & \ddots & & \\
& 0 & & 0 & W_{k+1} & \\
& & & & \ddots & \ddots
\end{array}\right) \begin{gathered}
\mathbb{C}^{n} \\
\mathbb{C}^{n} \\
\vdots \\
\mathbb{C}^{n} \\
\vdots
\end{gathered}
$$

an (a unilateral backward) operator weighted shift with weight sequence $\left\{W_{k}\right\}_{k=1}^{\infty}$, denoted by $S \sim\left\{W_{k}\right\}$. Moreover, $n$ is called the multiplicity of $S$.

First, by a routine computation, we have the following.

Proposition 2.1. Let $S \sim\left\{W_{k}\right\}$ be an operator weighted shift. Then $T \in \mathcal{A}^{\prime}(S)$ if and only if $T$ is upper-triangular and

$$
T_{k k+l}=\left(W_{1} \cdots W_{k}\right)^{-1} T_{0 l} W_{l+1} \cdots W_{l+k}(k \geq 1, l \geq 0) .
$$

Obviously, $\operatorname{ker} S=\left\{\left(x_{k}\right) \in \mathcal{H}: x_{k}=0, \forall k \geq 1\right\}$. Set $\mathcal{A}_{0}(S)=\left.\mathcal{A}^{\prime}(S)\right|_{\text {ker } S}$. In 3, C. L. Jiang and J. X. Li proved the following.

Theorem 2.1. Let $S \sim\left\{W_{k}\right\}$ be an operator weighted shift. Then $S \in S I$ if and only if $\sigma\left(T_{0}\right)$ contains only one point for every $T_{0}$ in $\mathcal{A}_{0}(S)$.

\section{The COnstruction of the COUnterexample}

We consider the operator weighted shift $S \sim\left\{W_{k}\right\}$ and let $W_{k}=\lambda_{k}+J_{2}$, where $0 \neq \lambda_{k} \in \mathbf{C}$ and $J_{2}$ is a $2 \times 2$ nilpotent Jordan block. A simple computation shows that

$$
W_{1} W_{2} \cdots W_{k}=\left(\begin{array}{cc}
u_{k} & u_{k}^{\prime} \\
0 & u_{k}
\end{array}\right)
$$


where

$$
\begin{aligned}
u_{k} & =\prod_{i=1}^{k} \lambda_{i}, \\
u_{k}^{\prime} & =\sum_{1 \leq i_{1}<i_{2}<\cdots<i_{k-1} \leq k} \lambda_{i_{1}} \lambda_{i_{2}} \cdots \lambda_{i_{k-1}} .
\end{aligned}
$$

Thus, $u_{k}^{\prime} u_{k}^{-1}=\sum_{i=1}^{k} \frac{1}{\lambda_{i}}$. Also, we have

$$
\left(W_{1} W_{2} \cdots W_{k}\right)^{-1}=\left(\begin{array}{cc}
v_{k} & v_{k}^{\prime} \\
0 & v_{k}
\end{array}\right)
$$

where $v_{k}=u_{k}^{-1}$ and $v_{k}^{\prime}=-u_{k}^{\prime} u_{k}^{-2}$.

Theorem 3.1. Let $S \sim\left\{W_{k}\right\}$ be given as above. Then $S \in S I$ if and only if

$$
\sup _{k}\left\{\left|\sum_{i=1}^{k} \lambda_{i}^{-1}\right|\right\}=\infty \text {. }
$$

Proof. Assume that (3.1) is false. Set $P_{0}=\left(\begin{array}{ll}1 & 0 \\ 0 & 0\end{array}\right)$. Then

$$
P_{k}:=\left(W_{1} W_{2} \cdots W_{k}\right)^{-1} P_{0} W_{1} W_{2} \cdots W_{k}=\left(\begin{array}{cc}
1 & v_{k} u_{k}^{\prime} \\
0 & 0
\end{array}\right) .
$$

Since $\left|v_{k} u_{k}^{\prime}\right|=\left|u_{k}^{\prime} u_{k}^{-1}\right| \leq \sup _{k}\left\{\left|\sum_{i=1}^{k} \frac{1}{\lambda_{i}}\right|\right\}<\infty$ we have $\sup _{k}\left\{\left\|P_{k}\right\|\right\}<\infty$. By Proposition 2.1, it follows that the nontrivial idempotent operator

is in $\mathcal{A}^{\prime}(S)$. Thus, $S \notin S I$.

$$
\operatorname{diag}\left(P_{0}, \cdots, P_{k}, \cdots\right)
$$

On the other hand, suppose that (3.1) is true. For every $T_{0}=\left(t_{i j}\right) \in \mathcal{A}_{0}(S)$, there exists an operator $T \in \mathcal{A}^{\prime}(S)$ such that $T_{0}=\left.T\right|_{\text {ker } S}$. Let the $k$ th diagonal element of $T$ be $T_{k}=\left(t_{i j}^{(k)}\right)$. By Proposition 2.1,

$$
T_{k}=\left(W_{1} W_{2} \cdots W_{k}\right)^{-1} T_{0} W_{1} W_{2} \cdots W_{k} .
$$

Hence, we have

$$
\left|t_{11}^{(k)}\right|=\left|v_{k} t_{11} u_{k}+v_{k}^{\prime} t_{21} u_{k}\right| \geq\left|u_{k}^{\prime} u_{k}^{-1}\right|\left|t_{21}\right|-\left|t_{11}\right| .
$$

Note that $\left|t_{11}^{(k)}\right| \leq\left\|T_{k}\right\| \leq\|T\|$; it follows that $t_{21}=0$. Thus, we have

$$
\left|t_{12}^{(k)}\right|=\left|v_{k} t_{11} u_{k}^{\prime}+v_{k} t_{12} u_{k}+v_{k}^{\prime} t_{22} u_{k}\right| \geq\left|u_{k}^{\prime} u_{k}^{-1}\right| \cdot\left|t_{11}-t_{22}\right|-\left|t_{12}\right| \text {. }
$$

Still, since $\left|t_{12}^{(k)}\right| \leq\|T\|$, we see that $t_{11}=t_{22}$. Thus $\sigma\left(T_{0}\right)$ contains only one point. By Theorem 2.1, it follows that $S \in S I$.

Now, we can give our example as follows.

Example 3.1. Let $\left\{\lambda_{k}\right\}_{k=1}^{\infty}$ be given by the sequence

$$
\{1,-1,2^{-1},-2^{-1}, \cdots, \overbrace{1,-1, \cdots, 1,-1}^{2 j},(j+1)^{-1},-(j+1)^{-1}, \cdots\} .
$$

Then (3.1) holds. It follows from Theorem 3.1 that $S \in S I$. Also, by Corollary 2.1 of [7] and Theorem 4 of [8], we obtain that $\sigma(S)=\mathbb{B}:=\{z \in \mathbb{C}:|z| \leq 1\}$. Set $A_{0}=\left(\begin{array}{ll}0 & 1 \\ 0 & 0\end{array}\right)$. Then

$$
A_{k}:=\left(W_{1} W_{2} \cdots W_{k}\right)^{-1} A_{0} W_{1} W_{2} \cdots W_{k}=A_{0} .
$$


Thus,

$$
A:=\operatorname{diag}\left(A_{0}, \cdots, A_{k}, \cdots\right) \in \mathcal{A}^{\prime}(S) .
$$

Next, set $B_{01}=\left(\begin{array}{ll}0 & 0 \\ 1 & 0\end{array}\right)$. It follows that

$$
\begin{aligned}
B_{k k+1} & :=\left(W_{1} W_{2} \cdots W_{k}\right)^{-1} B_{01} W_{2} W_{3} \cdots W_{k+1} \\
& =\left(\begin{array}{cc}
v_{k} & v_{k}^{\prime} \\
0 & v_{k}
\end{array}\right)\left(\begin{array}{cc}
0 & 0 \\
1 & 0
\end{array}\right)\left(\begin{array}{cc}
\bar{u}_{k} & \bar{u}_{k}^{\prime} \\
0 & \bar{u}_{k}
\end{array}\right)=\left(\begin{array}{cc}
v_{k}^{\prime} \bar{u}_{k} & v_{k}^{\prime} \bar{u}_{k}^{\prime} \\
v_{k} \bar{u}_{k} & v_{k} \bar{u}_{k}^{\prime}
\end{array}\right),
\end{aligned}
$$

where

$$
\begin{aligned}
\bar{u}_{k} & =\prod_{i=2}^{k+1} \lambda_{i}, \\
\bar{u}_{k}^{\prime} & =\sum_{2 \leq i_{1}<i_{2}<\cdots<i_{k-1} \leq k+1} \lambda_{i_{1}} \lambda_{i_{2}} \cdots \lambda_{i_{k-1}} .
\end{aligned}
$$

By calculating, we have that

$$
\begin{aligned}
\left|v_{k} \bar{u}_{k}\right| & =\left|\frac{\bar{u}_{k}}{u_{k}}\right|=\left|\lambda_{k+1}\right| \leq 1, \\
\left|v_{k}^{\prime} \bar{u}_{k}\right| & =\left|\frac{\bar{u}_{k}}{u_{k}} \frac{u_{k}^{\prime}}{u_{k}}\right|=\left|\lambda_{k+1} \sum_{i=1}^{k} \frac{1}{\lambda_{i}}\right| \leq 1, \\
\left|v_{k} \bar{u}_{k}^{\prime}\right| & =\left|\frac{\bar{u}_{k}^{\prime}}{u_{k}}\right|=\left|\lambda_{k+1} \sum_{i=2}^{k+1} \frac{1}{\lambda_{i}}\right| \leq 1, \\
\left|v_{k}^{\prime} \bar{u}_{k}^{\prime}\right| & =\left|\frac{u_{k}^{\prime}}{u_{k}} \frac{\bar{u}_{k}^{\prime}}{u_{k}}\right|=\left|\lambda_{k+1} \sum_{i=1}^{k} \frac{1}{\lambda_{i}} \sum_{i=2}^{k+1} \frac{1}{\lambda_{i}}\right| \leq 1 .
\end{aligned}
$$

Thus, $\sup _{k}\left\{\left\|B_{k k+1}\right\|\right\}<\infty$. Proposition 2.1 implies that

$$
B:=\left(\begin{array}{cccccc}
0 & B_{01} & & & & \\
& 0 & B_{12} & & 0 & \\
& & \ddots & \ddots & & \\
& & & 0 & B_{k k+1} & \\
& 0 & & & \ddots & \ddots
\end{array}\right) \in \mathcal{A}^{\prime}(S) .
$$

Let $T=A B-B A$. Then

$$
T_{01}=A_{0} B_{01}-B_{01} A_{1}=\left(\begin{array}{cc}
1 & 0 \\
0 & -1
\end{array}\right) .
$$

But

$$
T^{2} \equiv X=\left(\begin{array}{ccccccc}
0 & 0 & X_{02} & & & & \\
& 0 & 0 & X_{13} & & 0 & \\
& & \ddots & \ddots & \ddots & & \\
& & & 0 & 0 & X_{k k+2} & \\
& 0 & & & \ddots & \ddots & \ddots
\end{array}\right)
$$

where

$$
X_{02}=T_{01} T_{12}=T_{01} W_{1}^{-1} T_{01} W_{2}=\left(T_{01} W_{1}^{-1}\right)^{2} W_{1} W_{2}=W_{1} W_{2} .
$$

As a result, we obtain $T^{2}=S^{2}$. This implies that $\sigma\left(T^{2}\right)=(\sigma(S))^{2}=\mathbb{B}$. Thus $\sigma(T) \neq\{0\}$. So, $T \notin \operatorname{rad} \mathcal{A}^{\prime}(S)$. This indicates that $\mathcal{A}^{\prime}(S) / \operatorname{rad} \mathcal{A}^{\prime}(S)$ is not commutative. 
Remark. J. X. Li, Y. Q. Ji and S. L. Sun [7] proved that $S \sim\left\{W_{k}\right\}$ is a CowenDouglas operator if and only if $\sup _{k}\left\{\left\|W_{k}^{-1}\right\|\right\}<\infty$. Clearly, $S \sim\left\{W_{k}\right\}$ in Example 3.1 is not a Cowen-Douglas operator.

\section{REFERENCES}

[1] M. J. Cowen and R. G. Douglas, Complex geometry and operator theory, Acta Math. 141 (1978), 187-261. MR501368 (80f:47012)

[2] C. L. Jiang, Similarity classification of Cowen-Douglas operators, Canad. J. Math. 56 (2004), 399-413. MR2074045 (2006d:47037)

[3] C. L. Jiang and J. X. Li, The irreducible decomposition of Cowen-Douglas operators and operator weighted shifts, Acta Sci. Math. (Szeged) 66 (2000), 679-695. MR.1804217(2001m:47059)

[4] C. L. Jiang and Z. Y. Wang, Strong irreducible operators on Hilbert space, Pitman. Res. Notes Math. 389, Longman Harlow, Essex, 1998. MR.1640067 (2000c:47033)

[5] C. L. Jiang and Z. Y. Wang, Structure of Hilbert space operators, World Scientific Publishing Co. Pte. Ltd., 2006. MR2221863 (2008j:47001)

[6] A. L. Lambert, Unitary equivalence and reducibility of invertibly weighted shifts, Bull. Austral. Math. Soc. 5 (1971), 157-174. MR0295128 (45:4196)

[7] J. X. Li, Y. Q. Ji and S. L. Sun, The essential spectrum and Banach reducibility of operator weighted shifts, Acta Mathematica Sinica (English Series) 17 (2001), 413-424. MR.1852955 (2002g:47063)

[8] A. L. Shields, Weighted shift operators and analytic function theory, Topics in Operator Theory, Math. Surveys, Vol. 13, Amer. Math. Soc., Providence, RI, 1974, 49-128. MR0361899 $(50: 14341)$

School of Mathematics, Liaoning University, 110036, Shenyang, People's Republic of China

E-mail address: juexianli@sina.com 